\title{
Genèse du poème "Trahison fidèle » dans Stèles de Victor Segalen
}

Jean-Jacques Queloz et Danielle Terrien

\section{(2) OpenEdition}

1 Journals

Édition électronique

URL : https://journals.openedition.org/genesis/1317

DOI : 10.4000/genesis.1317

ISSN : 2268-1590

Éditeur :

Presses universitaires de Paris Sorbonne (PUPS), Société internationale de génétique artistique littéraire et scientifique (SIGALES)

Édition imprimée

Date de publication : 15 avril 2014

Pagination : 181-190

ISBN : 9782840509370

ISSN : $1167-5101$

\section{Référence électronique}

Jean-Jacques Queloz et Danielle Terrien, « Genèse du poème «Trahison fidèle » dans Stèles de Victor Segalen », Genesis [En ligne], 38 | 2014, mis en ligne le 27 juin 2016, consulté le 17 août 2022. URL : http://journals.openedition.org/genesis/1317 ; DOI : https://doi.org/10.4000/genesis.1317 


\title{
Genèse du poème "Trahison fidèle » dans Stèles de Victor Segalen
}

\author{
Danielle Terrien et Jean-Jacques Queloz
}

$\mathrm{L}$

e recueil Stèles que nous connaissons aujourd'hui résulte de plusieurs éditions.

En 1912, l'ouvrage qui paraît est composé de quarante-huit poèmes. Il n'était pas destiné à la vente et Segalen envoya personnellement les quatre-vingtun exemplaires ${ }^{1}$ que comptait le tirage à différentes personnalités du monde des lettres et des arts, ainsi qu'à des amis, parmi lesquels Henry Manceron ${ }^{2}$ qui suivait son travail depuis le premier jour.

Le 16 décembre 1913, Segalen fait paraître seize poèmes au Mercure de France, dont le texte intitulé "Trahison fidèle ». Ces poèmes seront ajoutés aux quarante-huit poèmes parus en 1912 et l'ensemble sera publié au début de 1914.

«Trahison fidèle » est le fruit d'une élaboration qui s'est déroulée par étapes successives. De fait, nous possédons plusieurs états du texte, ce qui autorise à adopter une perspective génétique, autrement dit à lire la version finale du poème à la lumière des versions qui l'ont précédée. Il s'agit d'une démarche qui ne peut être que féconde relativement à la poétique ségalénienne.

Il existe sept versions de ce poème. Elles constitueront notre dossier génétique (voir fig. 1 à 7). Segalen a pris soin de classer les différents feuillets et d'attribuer à chacun une lettre. Les trois premières versions sont chiffrées à l'aide de capitales $(\mathrm{A}, \mathrm{B}, \mathrm{C})^{3}$ alors que ce sont des minuscules qui désignent les quatre dernières $(\mathrm{d}, \mathrm{e}, \mathrm{f}, \mathrm{g})^{4}$.

Le lecteur du dossier génétique est d'emblée frappé par les faits suivants : la première version (A) ne comporte pas de titre ; les versions $\mathrm{B}, \mathrm{C}, \mathrm{d}$ sont intitulées «Trahison » alors que les trois dernières versions (e, f, g) ont pour titre «Trahison fidèle », une expression oxymorique qui ne fait qu'accroître plus encore la curiosité de ce lecteur. En outre, deux indications figurent sous le corps du poème (version $\mathrm{A}$ ), en bas à droite. Elles disparaitront des versions suivantes. Ces indications énigmatiques - méritent notre intérêt.

La première, "Henry $\mathrm{M}$ », renvoie à Henry Manceron. La seconde, "Chav. CCXXVII », fait référence au sinologue Édouard Chavannes, dont Segalen fut l'ami et le disciple. Considérons d'abord cette dernière et remettons-nous-en à Henry Bouillier qui, en 1982, dans son édition critique parue au Mercure de France, faisait les remarques suivantes :

On trouve en effet au tome I, à la page CCXXVII de l'Introduction [aux Mémoires historiques de Sseu-ma ts'ien] une note de Chavannes expliquant l'allusion à Po-ya faite par Sseu-ma ts'ien dans une lettre adressée à son ami Jen Ngan. La voici : " Tchong Tse-ki et Po-ya étaient tous deux du pays de Tch'ou. Po-ya jouait admirablement du luth. Tchong Tse-ki

1. « Il a choisi ce nombre de quatre-vingt-un parce que celui-ci est, dans la tradition chinoise, un nombre remarquable : c'est le carré du chiffre neuf, symbole à la fois du Ciel et de l'empereur, et c'est aussi, dans l'enceinte du Temple du Ciel, au sud de Pékin, le nombre de dalles disposées en cercles concentriques sur la terrasse supérieure du grand tertre rond, construit en marbre blanc », Trahison fidèle. Correspondance (19071918), rassemblée, présentée et annotée par Gilles Manceron, Paris, Éditions du Seuil, 1985, p. 125. Gilles Manceron est le petit-fils de Henry Manceron.

2. Henry Manceron figure à la sixième place de la liste des quatrevingt-un destinataires. La première place est occupée par Paul Claudel auquel le recueil Stèles est dédié

3. BnF, NAF 25817, mf 3784, fos 25, 26, 27.

4. BnF, NAF 25817, mf 3784, fos $28,29,30,31$. 
goûtait fort sa musique et devinait, en l'entendant, ses pensées. Quand il mourut, Po-ya, ayant perdu celui qui seul savait l'apprécier, cessa de jouer. » Ce texte est évidemment la source directe du poème ${ }^{5}$.

L'allusion au luth de Po-ya et à sa musique6, que l'on retrouve dans plusieurs versions du texte de Segalen, à commencer par la première, atteste - si besoin était que le référent chinois est à l'origine du poème. Segalen s'intéressait non seulement aux ouvrages littéraires chinois mais aussi aux textes écrits par les Européens sur la littérature chinoise. Du reste, le poète n'a cessé d'être fasciné par les grands textes et les beaux livres.

Portons à présent notre attention sur l'autre indication figurant au-dessous du poème. Les enjeux sont ici beaucoup plus personnels puisque « Henry $\mathrm{M}$ » désigne l'ami de toujours de Segalen, un ami dont l'importance a été déterminante sur les choix de vie de l'auteur de René Leys.

En effet, c'est à l'écoute des récits de Manceron, qui l'a précédé en Chine en tant qu'officier de marine, que Segalen décide d'apprendre le chinois afin de devenir élève-interprète. Il pourra ainsi passer deux ans en Chine, détaché de la marine. À ce rôle de mentor exercé par Manceron s'ajoute également celui de critique. Et c'est ici, précisément, que l'indication figurant au bas du texte prend tout son sens. De fait, durant l'année 1911, Manceron lit et critique avec acuité les poèmes de Stèles déjà élaborés. Toutefois, en août 1912, Segalen ayant achevé le recueil, l'ayant dédicacé et envoyé à Manceron, ce dernier ne réagit pas. Segalen s'en inquiète dans une lettre datée du 29 décembre. Il ne comprend pas que l'ami qui a suivi jusque-là, avec beaucoup d'intérêt, son travail, ne lui ait pas fait part de ses impressions. Le 17 janvier 1913, Manceron écrit enfin à Segalen et lui explique les raisons de ce retard.

Tamaris, 17 janvier 1913

Mon cher Victor, ta lettre du 29 décembre me trouve aujourd'hui à Tamaris, où, avec Riquette et mes deux petits en parfaite santé, j'achève pour mon compte de me remettre de l'état de santé délabrée dans lequel je suis resté tout l'été après mon retour d'Indochine. Et ta lettre, comme l'appel entendu d'une voix familière et proche, secoue en sursaut la torpeur qui paralysait ma plume. Il faut que je te réponde de suite : je suis là, fidèle à l'écho de ta voix, attentif aux suggestions de ta pensée, ardemment fraternel à toutes les émotions et les fluctuations de ta vie.

\begin{abstract}
« Des lointains, des si lointains j'accours, ami, vers toi le plus cher. Mes pas ont dépecé l'horrible espace entre nous7. » Ai-je passé de longs mois sans t'écrire ? Stupide paralysie de ma plume. Tu me connais, taciturne, inexprimé, mais fidèle et inchangé8.
\end{abstract}

C'est moins les raisons de ce retard que certains éléments ${ }^{9}$ qui entreront dans la composition de la stèle «Trahison fidèle » que nous retiendrons. Dans une lettre datée du 11 juin 1913, Segalen explique à son ami comment cette stèle a vu le jour.

Le mécanisme déclenché, je n'ai pu faire autrement que l'écrire et la voici. Elle est encore très imparfaite, mal balancée ; - elle n'a pour moi que le mérite d'inclure, d'incorporer une parcelle de ta pensée, toute crue, toute vivante, à la mienne, et de reconnaître ainsi, mieux que par une dédicace, tout ce que je te dois d'immédiat et de bon ${ }^{10}$.

« Incorporer une parcelle de [...] pensée toute crue, toute vivante », n'est-ce pas précisément ce que fait Segalen lorsqu'il se sert d'expressions (« fidèle à l'écho de ma voix », « taciturne », « inexprimé », « fidèle ») dont Manceron avait usé dans sa lettre du 17 janvier ?

En outre, la date (mars 1913) qui figure en bas à gauche sous le poème indique que le texte a été composé environ deux mois après la réception de la lettre et atteste, si besoin était, la vraisemblable ascendance de la lettre de Manceron sur le poème qui sera intégré à l'ensemble « Stèles face au nord 11 », une section tout

5. Cité par Henry Bouillier, édition critique de Stèles, Paris, Mercure de France, 1982, p. 114.

6. Le nom de Po-ya va disparaître à partir de la quatrième version, ce qui marque, de la part de l'auteur, un recul par rapport au référent chinois et conséquemment dote le texte d'une portée plus universelle.

7. Dans une note, Gilles Manceron commente : «Henry Manceron cite les premières lignes de la stèle "Des lointains" ", dans Trahison fidèle. Correspondance (1907-1918), op. cit., p. 126.

8. Ibid.

9. Soulignés par nous.

10. Trahison fidèle. Correspondance (1907-1918), op. cit., p. 152-153. Segalen envoie à Manceron la septième version ( $\mathrm{g}$ ) de « Trahison fidèle ». 11. La section «Stèles face au midi » est dédiée aux décrets impériaux, «Stèles face au nord » à l'amitié, « Stèles orientées » à l'amour, « Stèles occidentées » à la guerre. À ces quatre directions-points cardinaux, Segalen en ajoute deux : le bord du chemin, pour lequel il écrira « Stèles du bord du chemin » et « Stèles du milieu », dans lesquelles il est question de « cet autre » qui est « moi ». 
Ami, tru es là, fibile à l'écho de mavoir atteritipar mexprimé mure or la sou or fidete, mon lutt. C'est pour toi geul que je four. chen

Ne t'en vas povit. Ne t'e cartes point. he me dis pas qu' if $x$ pounail qu'm jour tor n'enterdises pas. On bren qu, l'enterioant, tu ne devine plus le bon exat or ma pensé 1 d ...

Gur cela prisn venir! - Ne le ois par. Cor I' affurm alors, eprounant non luts les mains b'ares, l'ovilh áux remies son fiami les banches \& la

Imiler Po.ya, Jam son denil as micat \& oy ant perre celm. láprour qui tent il rowail foure, - viru Jusomais Jans le Ji. lenu.

Mars 19/3

Hemy $M \times$ char.

$$
\text { cexpvil }
$$

Fig. 1 : Victor Segalen, manuscrit de « Trahison fidèle », version A (BnF, NAF 25817, mf 3784, fo 25$)$

183 


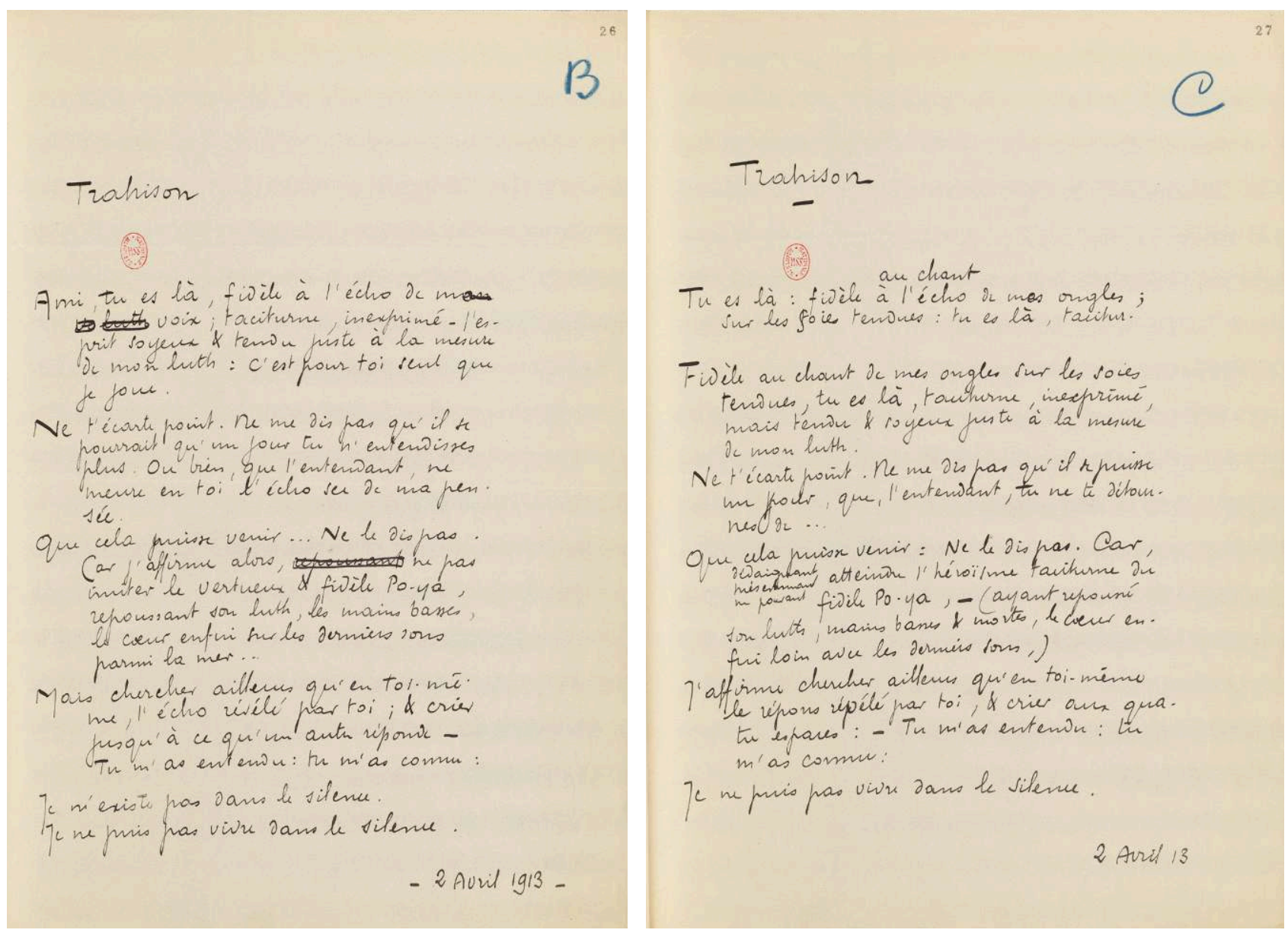

Fig. 2 et 3 : Victor Segalen, manuscrit de « Trahison fidèle », version B et C (BnF, NAF 25817, $\mathrm{mf} 3784, \mathrm{f}^{\circ} 26$ et f $\left.\mathrm{f}^{\circ} 27\right)$

entière consacrée à l'amitié. Et c'est bien d'amitié qu'il est question ici. Amitié sans faille pour Po-ya vis-à-vis de Tchong-Ki jusqu'à ce que la mort les sépare et plonge Po-ya dans le silence ; amitié entre Segalen et Henry Manceron en butte aux distances spatiale et temporelle.

L'importance du thème de l'amitié justifie donc une attention particulière et nous verrons qu'il n'est pas sans subir des altérations, aussi bien quant à la notion qu'il recouvre que quant aux formes de sa manifestation, au fil des différentes étapes qui conduisent à la version ne varietur du poème.

De fait, si dans la première version le poète veut imiter Po-ya, à savoir qu'il désire se réfugier dans le silence en cas d'éloignement éventuel de son ami, dans la seconde (B), il affirme vouloir « chercher ailleurs » et « crier jusqu'à ce qu'un autre réponde ». La fin du poème, « Je n'existe pas dans le silence / Je ne puis vivre dans le silence », met l'accent sur l'impossibilité psychique et existentielle de Segalen. Le constat premier est suivi d'une affirmation traduisant l'impossibilité " à vivre dans le silence ». Le poète a besoin d'échanger, de communiquer pour vivre mais également pour écrire ; et le poème a besoin d'être lu pour exister, semble nous signifier Segalen. Que serait en effet une œuvre écrite sans lecteur?

En outre, le changement d'attitude à l'égard de Po-ya trouve un écho supplémentaire. De fait, le référent chinois que représente Po-ya est un élément empreint de tradition ; renoncer à son imitation et vouloir « chercher ailleurs ", c'est aussi, pour Segalen, une manière de confesser un besoin de nouveauté esthétique. La poésie n'a pas pour tâche d'imiter, de copier, de reproduire l'ancien mais de nous mettre face à quelque chose de nouveau.

Cette deuxième version propose donc une rupture importante d'avec la première, tant esthétique qu'existentielle. Cependant, cette version surprend par son intitulé, qui semble en porte-à-faux avec ce dont il est question ; d'autant plus que dans la troisième version l'apostrophe «Ami », qui servait d'ouverture 

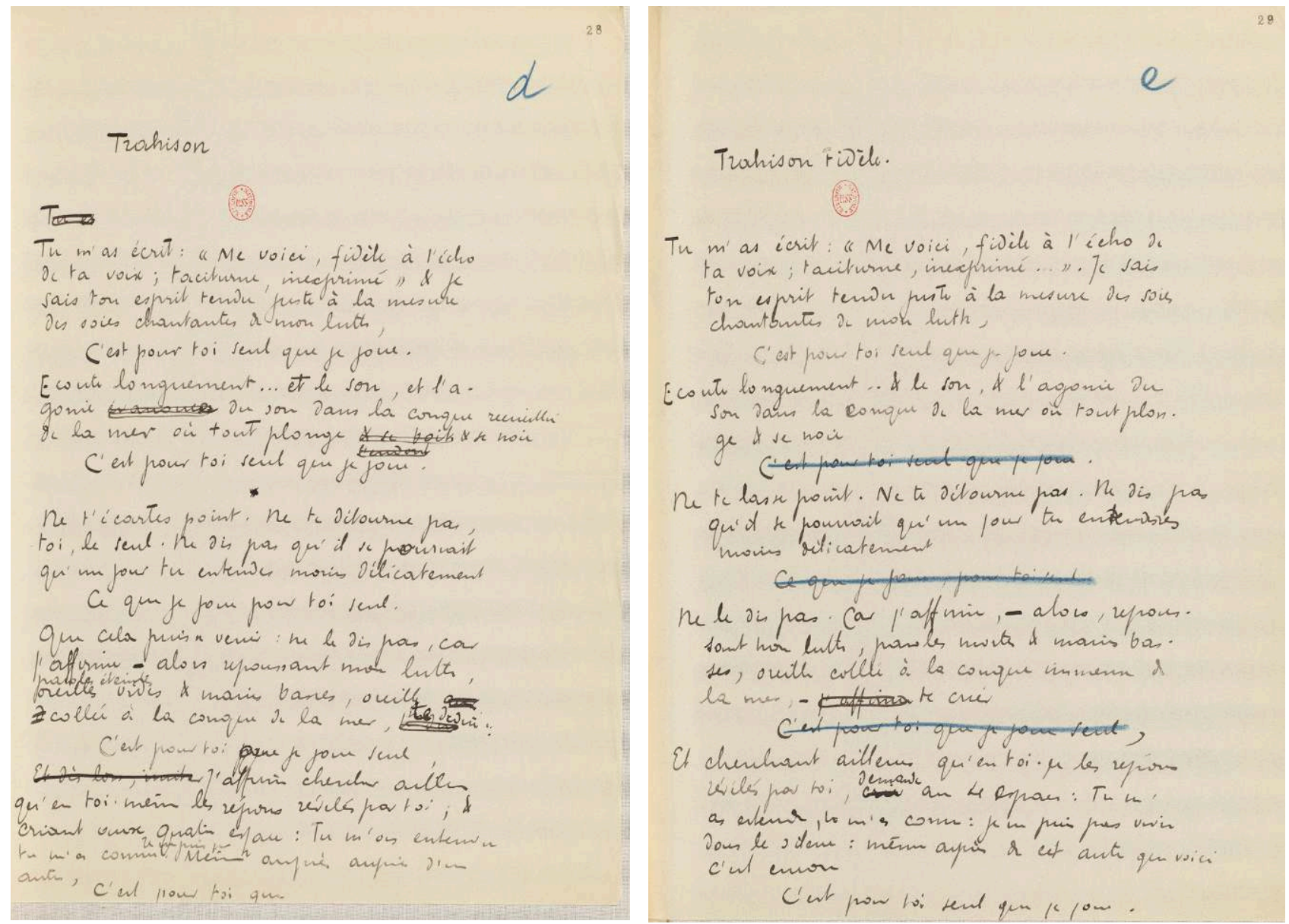

Fig. 4 et 5 : Victor Segalen, manuscrit de « Trahison fidèle », version d et e (BnF, NAF 25817, mf 3784, fo 28 et fo 29)

au poème et qui, d'une certaine manière, donnait le ton, a disparu. L'attitude du poète par rapport à la notion d'amitié est quelque peu déroutante : il l'évoque comme éminemment positive en même temps qu'il laisse filtrer une réserve certaine à son égard. Il faut attendre la cinquième version pour que l'amitié, indirectement, soit dotée d'un attribut positif et ce, grâce au titre oxymorique qui sera définitif. L'oxymore permet à Segalen de dire à la fois la fidélité et son manquement, une manière également d'indiquer que l'amitié n'est pas à l'abri de modifications, notamment parce que les amis peuvent changer. En témoigne la transformation suivante :

\section{Versiond}

[...] Même auprès auprès $[$ sic $]$ d'un autre,

Version e $\quad[\ldots]$ même auprès de cet autre que voici c'est encore

Versionf $\quad[\ldots]$ même auprès de ces autres que voici, c'est encore :
Versiong Même auprès de ces autres que voici, C'est encore

Version finale Même auprès de cet autre que voici, c'est encore,

Il s'agit ici de Jean Lartigue, que Segalen rencontre à Pékin, en 1912. Les deux hommes vont nourrir une intense amitié et Lartigue fera partie de la mission de 191412. L'ami peut toutefois devenir un étranger mais il peut aussi, au cœur de cette altérité, proposer de nouvelles similitudes, comme l'écrit Jean-Pierre Richard13 dans son article consacré à Stèles. Il peut également transmettre cette haute idée de l'amitié, d'ami en ami, comme c'est le cas dans «Trahison fidèle».

12. La mission Segalen-Lartigue-Voisins doit parcourir la Chine suivant une « Grande Diagonale », du nord-est au sud-ouest. Le but est double : archéologique et géographique. Elle sera interrompue par l'annonce de la guerre. 13. Jean-Pierre Richard, "Espaces stellaires », dans Pages Paysages. Microlectures II, Paris, Éditions du Seuil, coll. « Poétique », 1984, p. 131. 

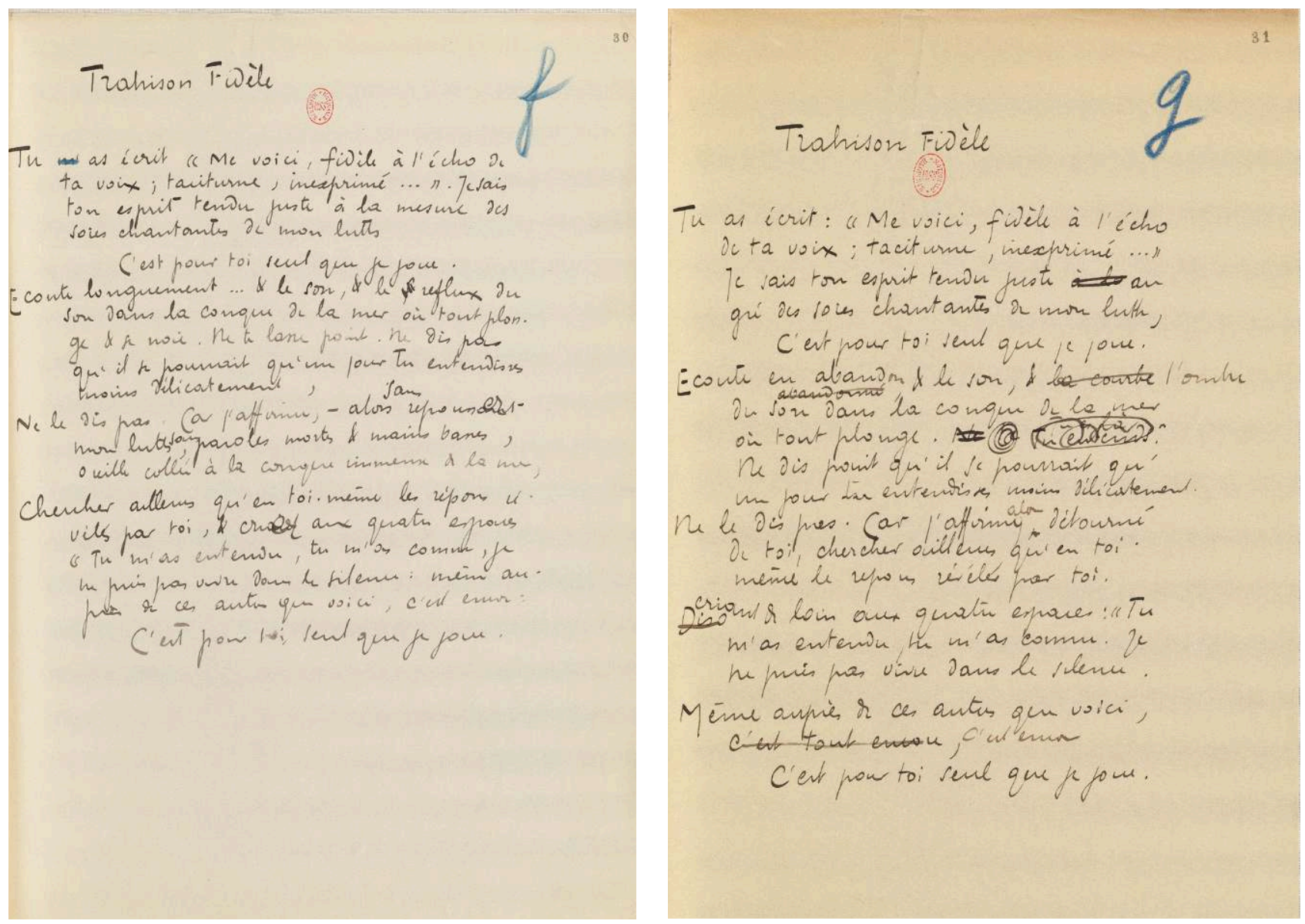

Fig. 6 et 7 : Victor Segalen, manuscrit de « Trahison fidèle », version $\mathrm{f}$ et $\mathrm{g}(\mathrm{BnF}, \mathrm{NAF} 25817, \mathrm{mf}$ 3784, fo 30 et fo 31$)$

En relation à la notion d'amitié, les diverses versions révèlent ce que l'on pourrait nommer des «questionnements », des "interrogations » de la part de l'auteur, qui sont en fait à l'image des changements à la merci desquels est placée l'amitié. Toutefois, quelles qu'aient été les hésitations, l'auteur imprime à sa démarche un mouvement bien défini grâce à l'épigraphe dont le sens est «En quête d'un écho amical14 ». Cette épigraphe qui n'apparaît que dans la version finale (fig. 8) donne un éclairage à l'ensemble du texte.

Considérons à présent le poème sur un plan plus formel. Les trois premières versions du poème sont monologiques. C'est-à-dire que le poète intègre les propos de Manceron à son propre discours. En outre, il interpelle et exhorte son ami (voir les injonctions de la deuxième strophe). En revanche, dans la quatrième version, grâce au discours direct, Segalen confère à son texte un aspect dialogique (« Tu m'as écrit : "Me voici fidèle à l'écho / de ta voix ; taciturne, inexprimé" »). Il modifie certes quelque peu le texte de la lettre de Manceron en rapportant le discours de ce dernier ${ }^{15}$, mais l'esprit en reste le même. Ainsi, il établit une distance entre celui dont il rapporte les propos et lui-même, distance qui est loin d'être négative, puisqu'elle va placer le « je » et le «tu »dans une situation leur permettant de dialoguer. En d'autres termes, cette distance va permettre au « je » de trouver un écho, de ne «pas vivre dans le silence ». Cette volonté de séparer les instances énonciatrices était déjà observable dans la troisième version où, peu avant la fin du texte, «Tu m'as entendu : tu m'as connu » se démarque de ce qui précède au moyen d'un tiret qui va être remplacé par

14. Traduction de Chou Ling. Le fait de ne pas traduire l'épigraphe ajoute au désir d'exotisme et d'opacité propres à l'entreprise de Segalen dans ce recueil.

15. «Je suis là » est devenu « Me voici ». « Fidèle à l'écho de ta voix » est identique. «Attentif aux suggestions de ta pensée » est devenu « et je sais ton esprit tendu juste à la mesure des soies chantantes de mon luth ». 


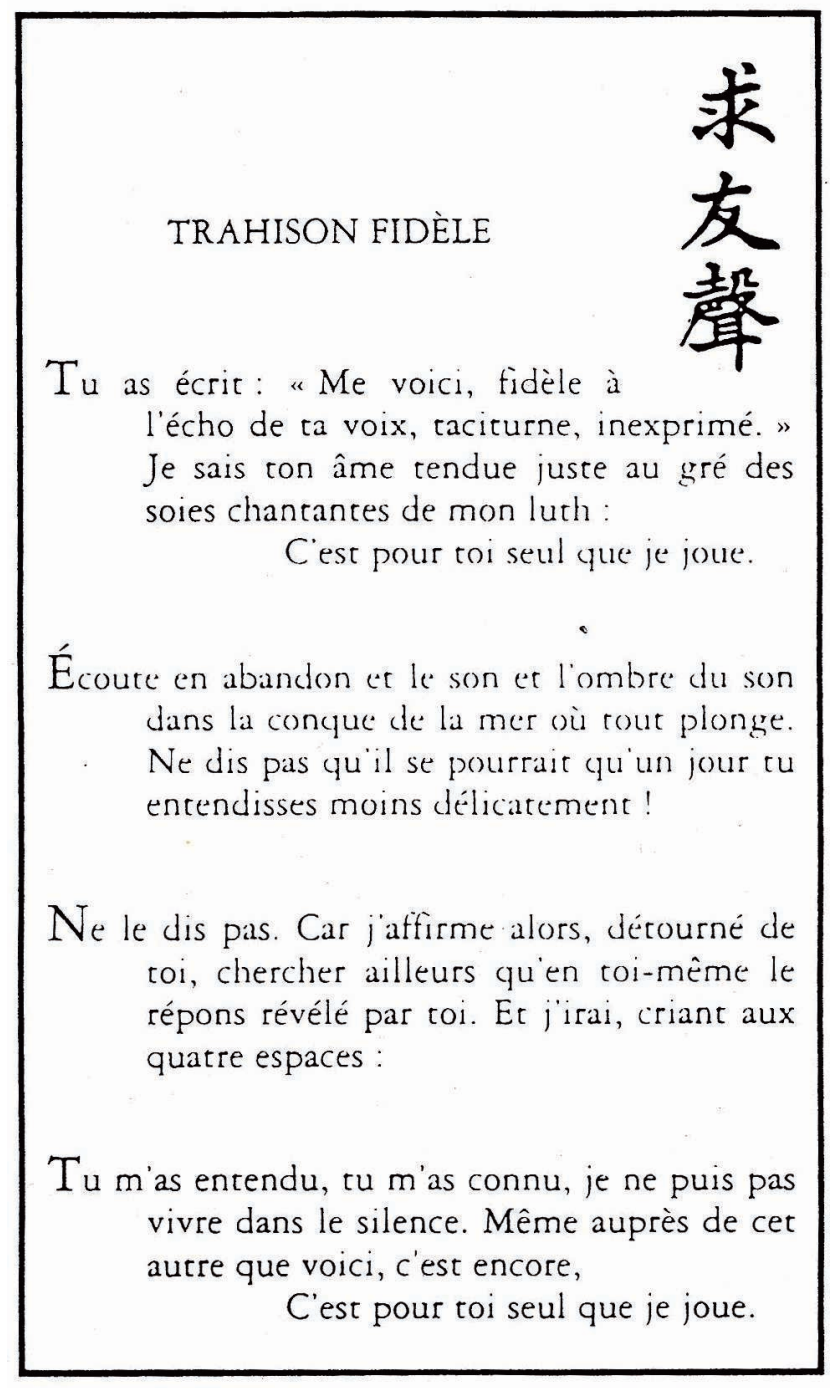

Fig. 8 : Version finale du poème (Paris, LGF, « Le Livre de poche », 1999, p. 129)

deux points dans la quatrième version et par des guillemets ouvrants dans la sixième et la septième versions. Le poète ne rapporte pas les propos d'autrui - comme c'est le cas au début du texte - certes, toutefois, que ce soit au moyen du tiret, des guillemets ou des deux-points, il confère au texte un caractère dialogique dont il faut tenir compte. Ce sont les deux-points qui vont clore la troisième strophe de la version finale et servir de signe démarcatif d'ouverture de la quatrième et dernière strophe du poème, celle-ci contribuant tout entière à inscrire le poème dans un mode d'énonciation qui est celui du dialogue.

L'aspect dialogique du poème qui apparaît sur le plan formel est essentiel puisqu'il est en étroite relation avec la thématique du texte à laquelle nous avons prêté attention plus haut, à savoir l'amitié. De fait, une partie du propos de la lettre de Manceron est incorporée dans le poème, tout comme ce que l'on pourrait nommer la mise en scène de l'aspect dialogique de la lettre. Cette mise en scène infléchit le poème du côté de l'échange, du partage, de la réciprocité. De la même manière que Manceron s'adresse au « tu », destinataire et ami qu'est Segalen, le poète accorde à son interlocuteur une place essentielle. Le monologue inquiet du début de la première version fait très vite place au dialogue revendiqué comme nécessité absolue et qui se doit d'être élargi par un subtil transfert d'amitié à l'autre, l'ami, le nouvel ami, et par extension, le lecteur en général. Le « je » lyrique s'adresse à un interlocuteur-destinataire singulier dans un premier temps, mais qui a tôt fait de devenir universel. Et c'est bien sûr aussi de l'activité poétique qu'il est question à travers la métaphore musicale, la musique renvoyant au poème, le luth (instrument) à la voix du poète et le 
corps du musicien à celui du poète. Toutefois, si la poésie est évoquée par le biais de la musique, le poète veut également une poésie qui soit musicale. En témoigne l'exemple suivant :

Écoute en abandon et le son et l'ombre du son dans la conque de la mer où tout plonge.

Segalen semble avoir mis une attention toute particulière à jouer sur le caractère allitératif de certains phonèmes comme « on » (abandon/son/ombre/conque/plonge) et à le mêler à d'autres, par exemple « ou » (écoute/où/ tout). En outre, un legato provoqué par les « et le16» à proximité des voyelles liquides « $\mathrm{e}^{17}$ » n'est sans doute pas accidentel. Ne rappelle-t-il pas au lecteur un lieu où l'écoute est absolue, un lieu chaud comme l'intérieur du pavillon de l'oreille, un espace plénier ? Une lecture « musicale »du poème «Trahison fidèle » serait à envisager; tel n'est pas notre propos ${ }^{18}$.

Nous souhaiterions enfin évoquer un dernier élément par lequel le texte de la version publiée se distingue des versions qui précèdent. Il s'agit, sur le plan typographique, du cadre rectangulaire dans lequel le poème est inscrit. Comment ne pas y voir une manière de clôture du poème par rapport à ses avant-textes 19 ?

Ce rectangle noir a une valeur d'autant plus emblématique que Segalen est sensible à la notion d'écriture en devenir. Le fait d'avoir numéroté et classé les différents états du texte en est la preuve. Nous pourrions être tentés de penser que l'encadré du poème est une manière de signifier un aboutissement du processus durant lequel le poète n'a cessé de modifier son texte. Nous avons relevé quelques exemples de ces modifications. Nous aurions pu en ajouter d'autres, tel le «C'est pour toi seul que je joue » qui, avant d'apparaître ainsi à la fin de la première et de la dernière strophe du poème, dès la version (e), a connu plusieurs formes, notamment dans la quatrième version (d) 20 .

Comme exemple de recherche de l'expression juste, nous mentionnerons également le « crier aux quatre espaces » (version c) qui deviendra successivement « criant aux quatre espace »(d), « demande au 4 espaces » $(\mathrm{e})$, « crier aux quatre espaces » $(\mathrm{f})$, « criant de loin aux quatre espaces » (g) pour, dans la version finale, reprendre la forme initiale, toutefois intégrée différemment au texte 21 .

La version imprimée offre encore de nouvelles modifications 22 .

Si nous éprouvons quelques doutes sur la fonction de l'encadré marquant de manière inéluctable l'achèvement du texte, il est certain par contre que ce fin liséré noir reproduit visuellement, dans l'espace de la page, une stèle de pierre revêtue de ses inscriptions.

Les différents états du texte qui sont à l'origine du poème «Trahison fidèle » se sont révélés riches d'informations quant au poème lui-même. La lecture de ce dernier, à la lumière des versions qui l'ont précédé, ne pouvait s'avérer que féconde. En effet, c'est par elles qu'il nous a été donné de mieux comprendre un titre qui, de prime abord, était pour le moins énigmatique. Sans l'étude des diverses variantes - en particulier la première - il aurait été difficile de soupçonner l'existence et l'importance des deux sources autobiographiques qui ont directement inspiré l'écriture du poème, à la fois

16. De « et le son et l'ombre ».

17. écoute / conque / plonge.

18. À titre d'exemple, nous mentionnerons l'expression : «C'est pour toi seul que je joue » et ses diverses variantes (voir note 21) qui, à cinq reprises, marque la fin des strophes (version d) à la manière d'un refrain. Enfin, est-il besoin de rappeler que Segalen était musicien ?

19. Selon Christian Doumet, « ce rectangle [...] impose une double clôture ». Voir Le Rituel du livre, Paris, Hachette supérieur, 1992, p. 38. 20. «C'est pour toi seul que je joue » / «Ce que je joue pour toi seul » / «C'est pour toi que je joue seul ».

21. Version (c)

J'affirme chercher ailleurs qu'en toi-même

le répons révélé par toi, \& crier aux qua-

tre espaces : - Tu m'as entendu : tu

m'as connu :

Version finale

$\mathrm{Ne}$ le dis pas. Car j'affirme alors, détourné de toi, chercher ailleurs qu'en toi-même le répons révélé par toi. Et j'irai, criant aux quatre espaces :

22. Nous allons donner deux exemples : les cinq séquences de la septième version manuscrite sont refondues en quatre. Dans la deuxième séquence de la septième version $(\mathrm{g}), \mathrm{le}$ 《 $\mathrm{Ne}$ dis point » de la quatrième ligne se transforme en « Ne dis pas », moins archaïsant dans la deuxième séquence, à la troisième ligne de la version imprimée (nous nous référons à l'édition du « Livre de poche », Paris, LGF, 1999, p. 129). 
éléments moteurs et nourriciers s'inscrivant au cœur de la trame textuelle. Elles mettent l'accent notamment sur la nécessité de l'amitié, thème central du poème et en dévoilent les multiples facettes.

Les versions successives du texte nous ont permis également de prendre conscience de certains procédés d'écriture chez Segalen, à travers la transformation et l'intégration d'un fragment de lettre par exemple.

De la même manière, la recherche de «l'expression juste », l'hésitation que nous avons pu constater à l'examen des variantes, nous ont révélé un poète dont l'écriture est en élaboration constante. Segalen a en effet travaillé son poème jusqu'à la version définitive, l'amenant à plus de concision, cherchant à redonner au mot sa pleine densité, son énergie.

En d'autres termes, les différents états du texte nous ont permis d'assister aux choix opérés par le poète dans l'ordre des possibles. Ils nous ont conduits à mieux saisir la poétique de Segalen.

Cette persistance à vouloir trouver le mot juste, l'expression qui convient, participe d'une grande exigence qui le mène à la découverte de formes nouvelles. Et, c'est par elles que le lecteur - entendons également l'ami - pourra être interpellé. 
DAnielle Terrien est docteur en littérature française ; elle a consacré sa thèse à Victor Segalen. Elle fait partie du groupe « Genèse et autobiographie » de l'ITEM (CNRS-ENS). Elle s'est tout particulièrement intéressée aux journaux de voyage de Segalen.

danielleterrien@magic.fr

JEAN-JACQues Queloz est chargé de cours à l'Université de Bâle (Suisse). Il a publié une thèse de doctorat sur Michel Leiris (Pour une poétique de Michel Leiris, Paris, Champion, 1999) et a consacré un travail d'habilitation à l'œuvre de Philippe Soupault (Philippe Soupault : écriture de soi et lecture d'autrui, Louvain-la-Neuve, Academia, 2012), en particulier à ses aspects autobiographiques. Il est membre de l'équipe «Genèse et autobiographie » de l'ITEM (CNRS-ENS).

j-j.queloz@unibas.ch

\section{Résumés Genèse du poème "Trahison fidèle " dans Stèles de Victor Segalen}

Victor Segalen publie une première édition de Stèles en 1912. Elle comporte quarante-huit poèmes. Une deuxième édition augmentée de seize poèmes voit le jour au début de 1914. «Trahison fidèle » en fait partie. L'élaboration de ce poème s'est déroulée par étapes successives. En effet, outre le texte publié que nous connaissons, il existe sept versions différentes du poème, que Segalen est même allé jusqu'à classer, ce qui permet d'adopter une perspective génétique et de pratiquer une lecture qui s'avère éclairante quant au contenu du poème lui-même - à commencer par son titre. L'étude des variantes dévoile la charge autobiographique de ce poème ; elle permet aussi de saisir à quel point la notion d'amitié est centrale et complexe pour le poète ; elle met également en évidence des procédés d'écriture, autant d'éléments qui contribuent à définir la poétique de Segalen.

Victor Segalen published a first version of Stèles in 1912. This volume contains forty-eight poems. A second edition is published in 1914 with an addition of sixteen poems. "Trahison infidèle" figures in this revised edition. The production of this poem went through several stages. Indeed, in addition to the published text that we know, there are seven different versions of this poem, which Segalen even filed. This allows us to have a genetics perspective and to practice a reading approach that is enlightening for the content of the poem itself, starting with its title. The study of the variants unveils the autobiographic aspects of this poem, and it allows us to understand how much the notion of friendship is central and complex for the poet. It also highlights writing techniques. All these elements contribute to defining Segalen's poetics.

1912 veröffentlicht Victor Segalen eine erste Ausgabe von Stèles, die aus achtundvierzig Gedichten besteht. Eine zweite, um sechzehn Gedichte erweiterte Ausgabe erscheint anfangs 1914. „Trahison fidèle“ ist Teil davon. Die Ausarbeitung dieses Gedichtes ist in sukzessiven Phasen verlaufen. Neben dem bekannten veröffentlichten Text bestehen nämlich sieben weitere, unterschiedliche Versionen des Gedichts. Und Segalen ist sogar so weit gegangen, diese zu klassifizieren, was es zulässt, sie aus einer genetischen Perspektive zu betrachten und so Aufschlüsse über den Inhalt des Gedichts selbst zu erlangen - angefangen zum Beispiel mit seinem Titel. Die Untersuchung der Varianten deckt die autobiographische Ladung des Gedichts auf, ermöglicht zudem zu begreifen, in welchem Ausmaß der Begriff der Freundschaft für den Autor zentral und komplex ist und verdeutlicht gleichermaßen den Schreibprozess - lauter Elemente, die zur Definition von Segalens Poesie beitragen.
Víctor Segalen publica una primera edición de Stèles en 1912; ésta comporta cuarenta y ocho poemas. Una segunda edición aparece a comienzos de 1914, con dieciséis nuevos poemas, entre los cuales "Trahison fidèle". La elaboración de este poema se desarrolló en etapas sucesivas. En efecto, además del texto publicado que conocemos, existen siete versiones diferentes del poema -que fueron incluso clasificadas por Segalen-, lo que permite adoptar una perspectiva genética y practicar una lectura que se revela esclarecedora con respecto al contenido mismo del poema -empezando por su título. El estudio de las variantes pone de manifiesto la carga autobiográfica del poema y permite entender hasta que punto la noción de amistad es central y compleja para el poeta, revelando al mismo tiempo procedimientos de escritura; elementos todos que permiten definir la poética de Segalen.

Victor Segalen publica uma primeira edição de Stèles em 1912. Tem quarenta e oito poemas. Uma segunda edição surge no início de 1914, aumentada por dezasseis poemas, entre os quais "Trahison fidèle". O desenvolvimento deste poema ocupou fases sucessivas. Na verdade, além do texto publicado que conhecemos, existem sete versões diferentes do poema, que Segalen foi mesmo ao ponto de classificar, permitindo uma perspectiva genética e uma leitura esclarecedora quanto ao conteúdo do poema em si - começando pelo título. O estudo das variantes revela a carga autobiográfica do poema; permite também captar a que ponto a noção de amizade é central e complexa para o poeta; destaca finalmente a escrita processada, todos estes elementos ajudando a definir a poética de Segalen.

Victor Segalen pubblica nel 1912, sotto il titolo Stèles, Una prima edizione delle sue poesie, costituita da quarantotto testi. Una seconda edizione della raccolta segue nel ' 14 , accresciuta di nuove sedici liriche composte nei primi mesi di quell'anno. Tra questi componimenti poetici, si legge "Trahison fidèle", la cui elaborazione è tuttavia scandita in più fasi. Infatti, del testo pubblicato nella raccolta dall'autore, esistono altre sette diverse redazioni, che lo stesso Segalen ha persino pre-catalogato, le quali consentono, sfruttando il panorama del superstite diagramma genetico, di compiere una lettura illuminante della sua trama - a partire dal titolo. L'esame delle varianti redazionali testimonia la dimensione autobiografica della lirica; permettendo così da un lato (ed è l'elemento diciamo autobiografico) di cogliere quanto l'idea di amicizia sia per il poeta essenziale ed insieme complessa, dall'altro (ed è l'elemento tecnico-stilistico) di accertare i procedimenti di composizione, capaci di precisare la poetica di Segalen. 